\title{
Didymosphenia geminata (Lyngbye) M. Schmidt y Gomphoneis minuta (Stone) Kociolek \& Stoermer (Bacillariophyta), especies presentes en ríos de la VIII Región, Chile, formadoras de proliferaciones con aspecto muy similar
}

\section{Didymosphenia geminata (Lyngbye) M. Schmidt and Gomphoneis minuta (Stone) Kociolek \& Stoermer (Bacillariophyta), species living in rivers of the VIII Region, Chile, forming massive proliferations with similar appearance}

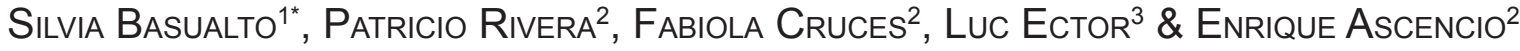 \\ ${ }^{1}$ Centro EULA, Universidad de Concepción, Casilla 160-C, Concepción, Chile. \\ ${ }^{2}$ Departamento de Botánica, Universidad de Concepción, Casilla 160-C, Concepción, Chile. \\ ${ }^{3}$ Luxembourg Institute of Science and Technology (LIST), Department Environmental Research and Innovation (ERIN), 41 rue \\ du Brill, L-4422 Belvaux, Luxembourg. \\ "sbasualt@udec.cl
}

\begin{abstract}
A light brown colored massive algal proliferation found in a large area of Rio Laja, Chile was studied. The bloom was produced by Gomphoneis minuta, a gomphonemoid diatom forming large branched stalks attached to submerged rocks. At first glance and on account the macroscopic appearance, this type of proliferation could be confused with that of Didymosphenia geminata. Considering its massive presence in Rio Laja and increased distribution in Chilean waters, as well is occurring in many European and American rivers, we postulate that G. minuta could be a nuisance species in Chilean rivers, and similar to D. geminata, it must be controlled to verify its propagation in the country.
\end{abstract}

Es frecuente que las diatomeas dominen los ambientes lóticos $\mathrm{y}$, como productores primarios, son esenciales para la cadena trófica del ecosistema acuático (Round et al. 1990). Algunos géneros de diatomeas del tipo cymbelloide y gomphonemoide producen pedúnculos mucilaginosos para adherirse a los sustratos rocosos de ríos, y la especie más conocida es Didymosphenia geminata (Lyngbye) M. Schmidt (Blanco \& Ector 2009). Estas masas mucilaginosas ocasionan la alteración de los ecosistemas acuáticos, que afecta a su estructura y función (Kilroy et al. 2009, Gillis \& Chalifour 2010, James et al. 2010) y disminuyen el valor estético (Spaulding \& Elwell 2007, Beville et al. 2012).

En el Hemisferio Sur la invasión de Didymosphenia geminata se ha caracterizado por ser una de las más agresivas en la historia reciente, y ha tenido un gran impacto ecológico y económico, principalmente por la velocidad de expansión y por el número de ríos afectados (Jaramillo et al. 2015). En Chile se encontró por primera vez a Didymosphenia geminata formando una floración en el río Espolón (alrededor de 44 $\mathrm{S}$ ) en el año 2010 (Segura 2011, Rivera et al. 2013). En los años siguientes se ha expandido hacia el norte a las regiones de Los Lagos $\left(40^{\circ} \mathrm{S}\right)$ y de Los Ríos (en $39^{\circ} \mathrm{S}$ ) así como también en Araucanía y Biobío, $38^{\circ} \mathrm{S}$ (Reid et al. 2012). En el transcurso de tres años, se ha confirmado la presencia de floraciones de esta especie en 11 cuencas en Chile y Argentina, abarcando más de 3.000 km desde la cuenca del río Biobío hasta el Río Grande en Tierra del Fuego (Leone et al. 2014).

El 16 de marzo de 2016 un sector amplio del Río Laja (37 18 '26"S-71'38'25'W) presentaba las piedras expuestas al torrente de agua casi completamente cubiertas por masas algales de color marrón claro, de $2-5 \mathrm{~cm}$ de espesor (Fig. 1). El estudio del material biológico recolectado, ingresado a la Colección de Diatomeas de la Universidad de Concepción bajo el número DIAT-CONC M-3485, y sus preparaciones permanentes DIAT-CONC 7699, 7700 y 7701, demostró la presencia de conglomerados macroscópicos formados por pedúnculos de mucílago ramificados dicotómicamente y unidos al sustrato rocoso. Los pedúnculos emergen desde el extremo basal de frústulos de diatomeas gomphonemoides con forma de cuñas en vista conectival (Fig. 2.A). Un centenar de individuos fueron medidos. Las valvas son grandes, 51-105 $\mu \mathrm{m}$ de largo y 17-23 $\mu \mathrm{m}$ de ancho, y su contorno varía entre formas claviformes-lanceoladas a espatuladas, heteropolares, con el extremo apical redondeado y no capitado, mientras que el extremo basal es 
más angosto y redondeado (Fig. 2. G-I). Las estrías (11-13 en $10 \mu \mathrm{m}$ ) son radiales en toda la valva, y están formadas por una doble hilera de diminutas perforaciones visibles en el microscopio fotónico con aumento de inmersión (100x). Existe un solo estigma en el área central, externamente de contorno circular. Una línea longitudinal se distingue en ambos lados de la valva, más cercana al margen que al centro valvar.

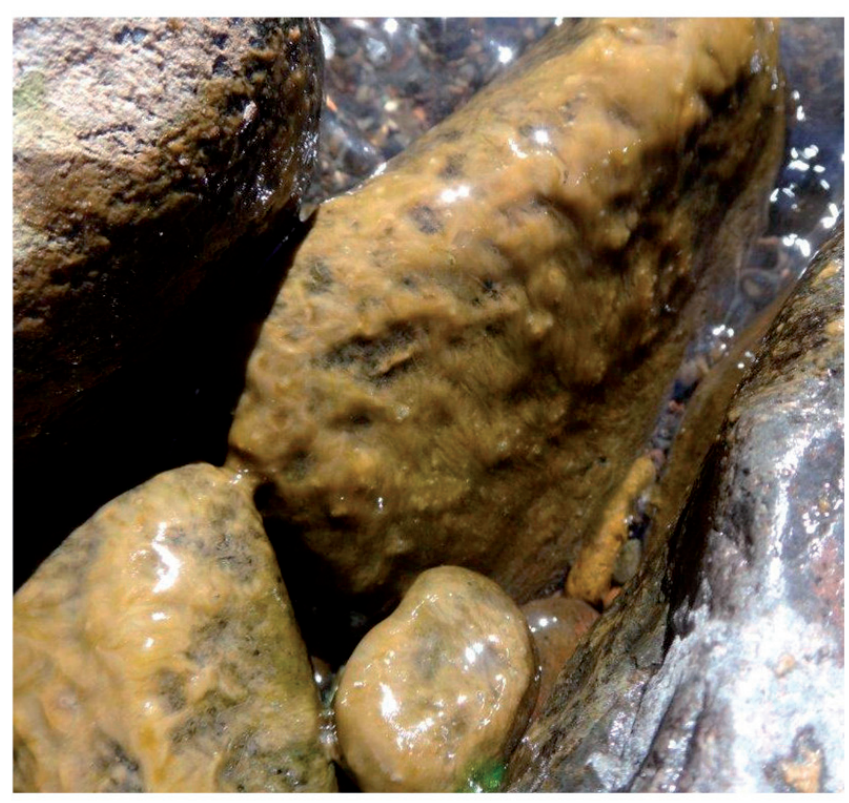

FIGURA 1. Aspecto general de las colonias de Gomphoneis minuta en el Río Laja.

FIGURE 1. General aspect of Gomphoneis minuta colonies at the Laja River.

Sobre la base de las características morfológicas señaladas, la diatomea encontrada en el Río Laja corresponde al género Gomphoneis Cleve, específicamente a G. minuta (Stone) Kociolek \& Stoermer, que según sus autores posee valvas de 20-112 $\mu \mathrm{m}$ de largo y 14-21 $\mu \mathrm{m}$ de ancho (Kociolek \& Stoermer 1988). Este es el primer reporte de la especie formando conglomerados masivos en aguas chilenas. La flora acompañante estaba constituida por escasos individuos de los géneros Diatoma Bory, Rhopalodia O. Müller, Navicula Bory, Encyonema Kützing, Nitzschia Hassall, Cocconeis Ehrenberg y Epithemia Kützing.

La prospección visual de un río con piedras de color marrón debido a la presencia de abundantes algas, podría llevar a pensar a un observador común en las proliferaciones que produce Didymosphenia geminata, apreciación que se vería fortalecida si se observan las características de los pedúnculos mucilaginosos y de los frústulos en forma de cuña bajo una lupa o un microscopio con aumento menor. Incluso la flora acompañante de ambas especies es muy similar. Sin embargo, D. geminata además de formar conglomerados macroscópicos de color marrón, también los presenta de color gris (moco de agua), sus valvas son de mayor tamaño (48-132 $\mu \mathrm{m}$ de largo y 25-45 $\mu \mathrm{m}$ de ancho según Metzeltin \& Lange-Bertalot (2014), y son nítidamente capitadas en ambos extremos, las estrías están formadas por una sola hilera de aréolas estructuradas diferentemente y están distribuidas en la parte central de la valva de manera alternadamente largas y cortas. Además, posee un mayor número de estigmas en el área central (2 a 5) con una morfología diferente.

Gomphoneis minuta presenta un amplio rango de tolerancia a las condiciones ambientales. Kociolek \& Stoermer (1988) la señalan en hábitats eutróficos y para aguas con alto contenido orgánico y/o alta conductividad y consideran que se desarrolla en ambientes recibiendo aportes orgánicos. Bey \& Ector (2013) indican que parece preferir aguas de $\mathrm{pH}$ alcalino y bien oxigenada, pero su ecología queda por definir en cuanto a la carga orgánica y los nutrientes. Mediante la transferencia de comunidades de diatomeas perifíticas desarrolladas sobre sustratos artificiales a partir de un sitio de referencia a un sitio contaminado, Gold et al. (2002) han comprobado que la abundancia relativa de G. minuta disminuye rápidamente cuando está expuesto a contaminación por metales pesados (cadmio y zinc). Por otra parte, en el sitio de estudio del Río Laja las condiciones ambientales corresponden a un ambiente oligotrófico. Este contrastante comportamiento ecológico ya ha sido señalado en la literatura para otros géneros y especies de diatomeas. Así por ejemplo, las especies Fragilaria crotonensis y Cyclotella comensis han sido señaladas en lagos con altos y bajos niveles de nutrientes (Tolotti et al. 2003, Saros et al. 2005, Goldenberg \& Lehman 2012, Kistenich et al. 2014).

Gomphoneis minuta es una especie que está siendo señalada, entre otros, para la flora de diversos países latinoamericanos, europeos y en Norteamérica. En América del Norte, Kociolek \& Stoermer (1988) y Johansen et al. (2004) han encontrado Gomphoneis minuta predominante en el curso inferior de los ríos y en lagos interiores, desde la Columbia Británica hasta Arizona, y del este hasta la costa atlántica. Frecuentemente está asociado con las colonias de Cymbella janischii (A.W. F. Schmidt) De Toni y $C$. mexicana (Ehrenberg) Cleve en la región del Noroeste del Pacífico (Bahls 2007). En Europa ha sido encontrada por primera vez en 1990 en Francia en el río Ardèche, afluente del río Ródano, cerca de Aubenas (región Auvernia-RódanoAlpes), después casi al mismo tiempo en el riachuelo de Nasbinals (Aubrac, Macizo Central, región LanguedocRosellón-Mediodía-Pirineos), en el río Garona (Toulouse, región Languedoc-Rosellón-Mediodía-Pirineos), en el río Tet (Olette, aguas arriba de Perpignan, región LanguedocRosellón-Mediodía-Pirineos), en el río Eyrieux (aguas abajo de Le Cheylard, región Auvernia-Ródano-Alpes), en el río Ariège (L'Hospitalet-près-l'Andorre, región Languedoc- 

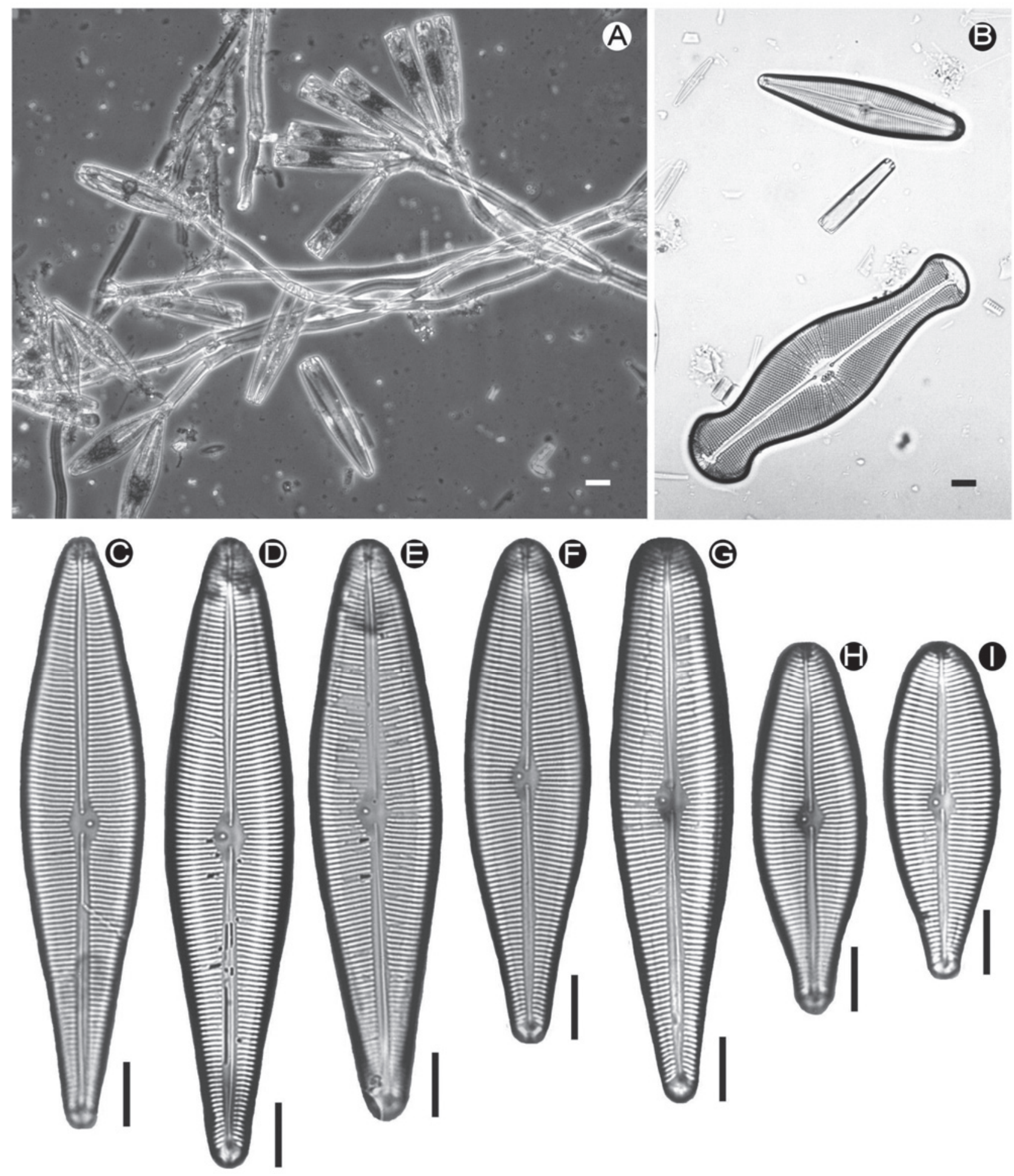

Figura 2. Gomphoneis minuta y Didymosphenia geminata. A. Pedúnculos de Gomphoneis minuta. Río Laja. B. Gomphoneis minuta (arriba) y Didymosphenia geminata (abajo). Río Biobío (Rivera et al. 2013). C-I. Variación del contorno valvar de G. minuta. Río Laja. Escalas: Fig. $\mathrm{A}=20 \mu \mathrm{m}$, Figs. B-I= $10 \mu \mathrm{m}$.

Figure 2. Gomphoneis minuta and Didymosphenia geminata, A. Gomphoneis minuta stalks. Laja River. B. Gomphoneis minuta (up) and Didymosphenia geminata (down). Biobío River (Rivera et al. 2013). C-I. Valve shape variation of G. minuta. Laja River. Scale bars: Fig. $\mathrm{A}=20 \mu \mathrm{m}$, Figs. B-I=10 $\mu \mathrm{m}$. 
Rosellón-Mediodía-Pirineos) y también más al norte en el río Loira (Orléans, región Centro-Valle de Loira), convirtiéndose en poco tiempo en una molestia para muchos ríos franceses, un país donde se la considera como una especie no indígena (Coste et al. 1992, Le Cohu \& Coste 1995, Coste \& Ector 2000, Blanco \& Ector 2009, Debenest et al. 2009, Bey \& Ector 2013). No obstante, G. minuta ha sido encontrada en 2013 mucho más al norte de Francia, en el río Doller, afluente del río Ill y del río Rin, en la región Alsacia-ChampañaArdenas-Lorena (Heudre et al. 2014). Gomphoneis minuta se ha extendido también en España, donde ha aparecido en algunos ríos del norte en la cuenca del río Ebro (Ortiz-Lerín et al. 2007) y en la cuenca del río Duero (Blanco et al. 2008, Kermarrec et al. 2011), a veces mezclado con las colonias de Didymosphenia geminata (por ejemplo en el río Omañas, León, en 2009 y 2010, Blanco, comunicación personal). En Portugal Novais (2011) ha señalado la presencia de G. minuta en agosto 2007 en el río Mosteiró (cuenca del río Duero o Douro). En Japón G. minuta también ha sido descubierto en el río Chikugo (Isla de Kyūshū, Suzawa et al. 2011), donde se une a las grandes colonias de Cymbella janischii utilizando su propio tallo de mucílago. En America del Sur, G. minuta ha sido señalado para Argentina por primera vez en 1989 y 1990 en el curso inferior del río Chubut en Patagonia (Ayestaran \& Sastre 1995). En Chile ha sido señalada desde el año 1971 para el Estero Lenga (Rivera et al. 1973), en los ríos Andalién y Biobío, desembocadura (Rivera 1974 y Rivera \& Arcos 1975), en los ríos Laraquete y Carampangue (Rivera \& Valdebenito 1979) y en la Bahía de Coliumo (Rivera \& González 1984). En estos trabajos se mencionan diferentes nombres de Gomphonema C. Agardh o Gomphoneis, que de acuerdo con la revisión del género hecha posteriormente por Kociolek \& Stoermer (1988) incluyendo los ríos chilenos Laraquete y Carampangue corresponden a G. minuta. La especie fue también señalada para los ríos Guaiquillo, VII Región, y Cautín, IX Región, por Rumrich et al. (2000). Además, al revisar el material estudiado por Rivera et al. (2013) correspondiente a la floración de $D$. geminata en la parte alta del río Biobío, IX Región de la Araucanía, comprobamos que G. minuta fue un componente de la flora acompañante del evento mencionado (Fig. 2 B). En los ríos Espolón, Coyhaique y Baker de la Región de Aysén, situados en el sur de Chile, Reid \& Torres (2014) han encontrado colonias localizadas de G. minuta en comparación con las floraciones de $D$. geminata.

Estimamos que sobre la base de su apariencia macroscópica las prospecciones visuales de Didymosphenia geminata podrían confundirse fácilmente con aquellas de Gomphoneis minuta. Además, el hecho que G. minuta se haya encontrado en forma masiva en el Río Laja y que su distribución en Chile se ha incrementado en el último tiempo, al igual que en ríos europeos y norteamericanos, nos hace presumir que sería capaz de transformarse en un taxón potencialmente nocivo para el ecosistema acuático, y que en forma similar a $D$. geminata, necesitaría de un monitoreo o programa de vigilancia para evitar su propagación.

\section{AGRADECIMIENTOS}

Agradecemos a dos revisores anónimos por sus sugerencias y comentarios al manuscrito. Proyecto de Investigación financiado parcialmente por la Vicerrectoría de Investigación y Desarrollo de la Universidad de Concepción.

\section{BIBLIOGRAFÍA}

Ayestaran, M.G. \& A.V. Sastre. 1995. Diatomeas del curso inferior del Río Chubut (Patagonia Argentina). Pennales I: Naviculaceae. Boletín de la Sociedad Argentina de Botánica 31(1-2): 57-68.

BAHLs, L.L. 2007. Cymbella janischii - giant endemic diatom of the Pacific Northwest: morphology, ecology and distribution compared to Cymbella mexicana. Northwest Science 81(4): 284-292.

Beville, S.T., G.N. Kerr \& K.F.D. Hughey. 2012. Valuing impacts of the invasive alga Didymosphenia geminata on recreational angling. Ecological Economics 82: 1-10.

Bey, M. \& L. Ector. 2013. Atlas des diatomées des cours d'eau de la région Rhône-Alpes. Tome 5. Naviculacées: Cymbelloidées, Gomphonématoidées. Direction Régionale de l'Environnement, de l'Aménagement et du Logement Rhône-Alpes, Lyon, pp. 751-971.

Blanco, S. \& L. Ector. 2009. Distribution, ecology and nuisance effects of the freshwater invasive diatom Didymosphenia geminata (Lyngbye) M. Schmidt: a literature review. Nova Hedwigia 88(3-4): 347-422.

Blanco, S., L. Ector, V. Huck, O. Monnier, H.M. Cauchie, L. Hoffmann \& E. BÉCARes. 2008. Diatom assemblages and water quality assessment in the Duero basin (NW Spain). Belgian Journal of Botany 141(1): 39-50.

Coste, M. \& L. Ector. 2000. Diatomées invasives exotiques ou rares en France: principales observations effectuées au cours des dernières décennies. Systematics and Geography of Plants 70(2): 373-400.

Coste, M., R. Le Cohu \& J. Bertrand. 1992. Sur l'apparition d'espèces du genre Gomphoneis en France. Distribution, caractéristiques morphologiques et écologiques. En: Agence de l'Eau Artois-Picardie (ed.), Actes du XIème Colloque des Diatomistes de Langue Française, Douai, 2427 Sept. 91, pp. 71-77. Agence de 1'Eau Artois-Picardie, Douai, France.

Debenest, T., J. Silvestre, M. Coste, F. Delmas \& E. Pinelli. 2009. A new cell primo-culture method for freshwater benthic diatom communities. Journal of Applied Phycology 21: 65-73.

Gillis, C.A. \& M. Chalifour. 2010. Changes in the macrobenthic community structure following the introduction of the invasive algae Didymosphenia geminata in the Matapedia River (Québec, Canada). Hydrobiologia 647(1): 63-70.

Gold, C., A. Feurtet-Mazel, M. Coste \& A. Boudou. 2002. Field transfer of periphytic diatom communities to assess shortterm structural effects of metals $(\mathrm{Cd}, \mathrm{Zn})$ in rivers. Water 
Didymosphenia geminata y Gomphoneis minuta formadoras de proliferaciones: BASUALTO, S. ET AL.

Research 36: 3654-3664.

Goldenberg, S. \& J. Lehman. 2012. Diatom response to the whole lake manipulation of a eutrophic urban impoundment. Hydrobiologia 691: 71-80.

Heudre, D., L. Moreau, L. Ector \& C.E. Wetzel. 2014. Arrivée et propagation d'espèces néobiotiques sur le bassin RhinMeuse: une première approche de 1997 à 2013. En: K. Serieyssol, A. Beauger \& L. Ector (eds.), $33^{\text {ème }}$ Colloque de l'Association des Diatomistes de Langue Française, 7-10 octobre 2014, p. 55. Programme et résumés des communications et des posters. Aubiere, France.

James, D.A., S.H. Ranney, S.R. Chipps \& B.D. Spindler. 2010. Invertebrate composition and abundance associated with Didymosphenia geminata in a montane stream. Journal of Freshwater Ecology 25(2): 235-241.

Jaramillo, A., D. Osman, L. Caputo \& L. Cardenas. 2015. Molecular evidence of a Didymosphenia geminata (Bacillariophyceae) invasion in Chilean freshwater systems. Harmful Algae 49: 117-123.

Johansen, J.R., R. Lowe, S.R. Gomez, J.P. Kociolek \& S.A. Masosky. 2004. New algal species records for the Great Smoky Mountains National Park, U.S.A., with an annotated checklist of all reported species for the park. Algological Studies 111:17-44.

Kermarrec, L., L. Ector, A. Bouchez, F. Rimet \& L. Hoffmann. 2011. A preliminary phylogenetic analysis of the Cymbellales based on 18S rDNA gene sequencing. Diatom Research 26(3): 305-315.

Kilroy, C., S.T. Larned \& B.J.F. Biggs. 2009. The nonindigenous diatom Didymosphenia geminata alters benthic communities in New Zealand rivers. Freshwater Biology 54: 1990-2002.

Kistenich, S., M. Drebler, J. Zimmermann, T. HüBener, R. Bastrop \& R. JAHN. 2014. An investigation into the morphology and genetics of Cyclotella comensis and closely related taxa. Diatom Research 29(4): 423-440.

Kociolek, J.P. \& E.F. Stoermer. 1988. Taxonomy, ultrastructure and distribution of Gomphoneis herculeana, G. eriense and closely related species (Naviculales: Gomphonemataceae). Proceedings of the Academy of Natural Sciences of Philadelphia 140(2): 24-97.

Le Cohu, R. \& M. Coste. 1995. Le genre Gomphoneis (Bacillariophyta) : un nouveau modèle d'organisation du cingulum. Canadian Journal of Botany 73: 112-120.

Leone, P.B., J. Cerda, S. Sala \& B. Reid. 2014. Mink (Neovison vison) as a natural vector in the dispersal of the diatom Didymosphenia geminata. Diatom Research 29(3): 259-266.

Metzeltin, D. \& H. Lange-Bertalot. 2014. The genus Didymosphenia M. Schmidt. A critical evaluation of established and description of 11 new taxa. Iconographia Diatomologica 25: 1-293.

NovaIs, M.H. 2011. Estudo das diatomáceas bênticas em sistemas lóticos de Portugal Continental. Benthic diatoms in Portuguese watercourses. Volume 2. Thesis Doutoramento em Biologia, Departamento de Biologia, Universidade de Évora, Évora, Portugal. 50 pp, 271 pls.

Ortiz-Lerín, R., C. Durán, M. Pardos \& J. Cambra. 2007. Red de diatomeas en la cuenca del Ebro: Evaluación de la calidad biológica del agua y estudio florístico de las comunidades durante el periodo 2005 y 2006. Algas 38: 10.

ReID, B. \& R. ToRres. 2014. Didymosphenia geminata invasion in South America: Ecosystem impacts and potential biogeochemical state change in Patagonian rivers. Acta Oecologica 54: 101-109.

Reid, B.L., K.L. Hernández, M. Frangópulos, G. Bauer, M. Lorca, C. Kilroy \& S. Spaulding. 2012. The invasion of the freshwater diatom Didymosphenia geminata in Patagonia: prospects, strategies, and implications for biosecurity of invasive microorganisms in continental waters. Conservation Letters 5: 432-440.

RiverA, P. 1974. Diatomeas de agua dulce de Concepción y alrededores, Chile. Gayana Botánica 28: 1-134.

Rivera, P. \& D. Arcos. 1975. Diatomeas más comunes en la desembocadura del Río Bio-Bío. Boletín de la Sociedad de Biología de Concepción 49: 223-230.

Rivera, P., S. Basualto \& F. Cruces. 2013. Acerca de la diatomea Didymosphenia geminata (Lyngbye) M. Schmidt: su morfología y distribución en Chile. Gayana Botánica 70(1): 154-158.

Rivera, P. \& M. GonzÁlez. 1984. Microalgas de los colectores de larvas de mitílidos en Bahía Coliumo, Chile. Phycologia Latino-Americana 2: 27-81.

Rivera, P., O. Parra \& M. González. 1973. Fitoplancton del Estero Lenga, Chile. Gayana Botánica 23: 1-93.

Rivera, P. \& H. Valdebenito. 1979. Diatomeas recolectadas en las desembocaduras de los ríos Chivilingo, Laraquete y Carampangue, Chile. Gayana Botánica 35: 1-99.

Round, F.E., R.M. Crawford \& D.G. Mann. 1990. The Diatoms. Biology \& Morphology of the Genera. Cambridge University Press, Cambridge. 747 pp.

Rumrich, U., H. Lange-Bertalot \& M. Rumrich. 2000. Diatomeen der Anden von Venezuela bis Patagonien/Feurland. Iconographia Diatomologica 9: 1-649.

Saros, J., T. Michel, S. Interlandi \& A. Wolf. 2005. Resource requirements of Asterionella formosa and Fragilaria crotonensis in oligotrophic alpine lakes: implications for recent phytoplankton community reorganizations. Canadian Journal of Fisheries and Aquatic Sciences 62: 1681-1689.

Segura, P. 2011. A slimy invader blooms in the rivers of Patagonia. Science 331: 18.

Spaulding, S.A. \& L. Elwell. 2007. Increase in nuisance blooms and geographic expansion of the freshwater diatom Didymosphenia geminata. Open-file report 2007-1425. U.S. Geological Survey, Reston. 38 pp.

Suzawa, T., S. Seino \& S. Mayama. 2011. Blooms of Cymbella janischii (A.W.F.Schmidt) De Toni accompanied by Gomphoneis minuta (Stone) Kociolek \& Stoermer from the upper stream of the Chikugo River, Kyushu, Japan, possibility of new alien diatom species. Diatom 27: 58-64.

Tolotti, M., H. Thies, M. Cantonati, C. Hansen \& B. Thaler. 2003. Flagellate algae (Chrysophyceae, Dinophyceae, Cryptophyceae) in 48 high mountain lakes of the Northern and Southern slope of the Eastern Alps: biodiversity, taxa distribution and their driving variables. Hydrobiologia 502: 331-348.

Recibido: 24.05.16

Aceptado: 10.10.16 